\title{
Time-dependent dynamical image screening at a metal surface
}

\author{
A. K. Kazansky ${ }^{1,2}$ and P. M. Echenique ${ }^{2,3}$ \\ ${ }^{1}$ V. A. Fock Institute of Physics, The University of St. Petersburg, St. Petersburg 198504, Russia \\ ${ }^{2}$ Donostia International Physics Center (DIPC), Paseo Manuel de Lardizabal, San Sebastián/Donostia, E-20018 Basque Country, Spain \\ ${ }^{3}$ Departamento de Fisica de Materiales, CFM, Centro Mixto CSIC-UPV/EHU, Apto 1072, San Sebastián/Donostia, \\ 20080 Basque Country, Spain
}

(Received 4 May 2010; published 28 May 2010)

\begin{abstract}
The energy loss by an electron ejected from a deep level of an atom adsorbed on $\operatorname{Ag}(110)$ surface is considered. It is shown that the dynamical effects in screening of the external field by a metal have to be taken into account and the energy loss by the ejected electron depends on the surface-plasmon dispersion and on the excitation energy. This effect can be observed with contemporary light sources.
\end{abstract}

DOI: 10.1103/PhysRevB.81.193413

PACS number(s): 73.20.At, 34.50.Bw, 73.90. +f

The subfemtosecond pulse technique ${ }^{1}$ initiated with the paper by Hentschel et $a l^{2}{ }^{2}$ has its aim in study of superfast relaxation processes caused by an abrupt change in the electron subsystem after an electron ejection. These investigations require development of theory, which provides deep understanding of the experimental findings. At the current level of computational technique, it seems to be possible to build up rather realistic models of the phenomena with atoms in the gas phase ${ }^{3}$ which make feasible, in principle, numerical control of the various aspects of the process. Application of subfemtosecond technique to solids is of great interest because a vast number of transient electron phenomena at surfaces and in solids does evolve on a few femtosecondsubfemtosecond time scale. Although the dynamics of electrons in condensed-matter systems is very complicated, its comprehensive understanding is necessary for development of experimental investigations and their application in nanotechnology. The experimental study of this dynamics has recently been performed. ${ }^{4}$ The computational simulations of the process have been presented in Refs. 5 and 6 .

In the processes with metal surfaces, the first feature to be discussed is formation of the response charge density in the metal and its influence on the ejected electron motion. In textbooks (see, e.g., Ref. 7), the interaction between a charged particle with the electric charge $q$ and a conducting plane surface is described with the potential energy $-q^{2} / 4 z$, where $z$ is the particle coordinate perpendicular to the surface ( $z=0$ at the metal image plane, $z$ is positive to vacuum). This potential energy has been obtained with the assumption of infinitely slow motion of a charged particle from infinity to the surface so that the electron system of the metal follows its motion adiabatically. However, when an atom adsorbed on a metal surface is ionized, the ejected electron does not move slowly. The energy loss by a charged particle moving with constant velocity at the vicinity of a metal surface has been addressed in a number of papers (see, e.g., Ref. 8 and the review, ${ }^{9}$ and references therein). Nevertheless, to our knowledge, electron motion with self-consistent account for its energy loss has not been addressed as yet. Here we consider this problem. At the first stage of the process, just after the electron ejection at the point $z_{0}$, the electron moves fast in the field of the adsorbate core and the induced metal charge distribution remains almost frozen. At this stage, the attraction of the electron by the induced charge distribution is greater than it is within the adiabatic treatment. Then, the initial image charge decays (oscillating with the surfaceplasmon frequency) while the field due to the dynamical screening of the moving electron arises (also with oscillations). At the initial stage, the attraction force is approximately $-\left(z+z_{0}\right)^{-2}$ (atomic units are used) and only after some time, which depends on the ejected electron energy and the surface-plasmon decay rate, the force becomes close to the value $-(2 z)^{-2}$. Thus, a theoretical consideration of the motion of an electron ejected from an object adsorbed on a metal surface requires a correct description of the time-dependent dynamical screening of its field by the metal.

Here we treat the metal response within the basic theory (Ref. 8 and references therein) and restrict ourselves with a very simple one-pole description of the relevant metalsurface dielectric function, taking its parameters from the $\mathrm{Ag}(110)$ experiments. ${ }^{10}$ (The surface plasmon on this surface is well pronounced and the corresponding plasmon frequency $\omega_{s p}=3.7 \mathrm{eV}$ is rather small.) The process under consideration is essentially nonlocal in time and its description implies solving the equation of motion of the electron selfconsistently with computation of the field acting on this electron, which depends on the entire previous history of its motion. For brevity, we consider only the case of onedimensional motion of an electron ejected from the adsorbate perpendicular to the metal surface.

Assume that an electron, being at rest for $t<0$ at the point $\vec{R}_{0}=\left(\vec{\rho}_{0}=0, z_{0}\right)$, is kicked by a photon absorption to the energy $E_{0}$. The basic equations describing the electron motion reads

$$
\begin{gathered}
\frac{d z_{1}}{d t}=v(t)=\sqrt{2\left\{E(t)-U_{c}\left[z_{1}(t)\right]-U_{c}^{i m}\left[z_{1}(t)\right]\right\}}, \\
\frac{d E}{d t}=v(t) F_{r}\left[z_{1}(t)\right], \quad F_{r}=\left.\frac{\partial}{\partial z} \phi_{r}(z, t)\right|_{z=z_{1}(t)}, \\
z_{1}(0)=z_{0}, \quad E(0)=E_{0} .
\end{gathered}
$$

Here $z_{1}(t)$ and $v(t)$ are the coordinate and the velocity of the ejected electron; $U_{c}(z), U_{c}^{i m}(z)$ the potentials of interaction of the electron with the adsorbate core and its time-independent electrostatic image [see Eq. (12)]; $F_{r}$ is the force acting on 
the moving electron from the charge density induced in the metal; $\phi_{r}(z, t)$ is the induced potential. In Eq. (1), $E(t)$ is the energy of the electron that does not include the contribution determined by the response of the metal, $E_{\infty}=E_{0}+A(\infty)$, where $E_{\infty}$ is the energy of the ejected electron and $A(\infty)$ $[A(\infty)<0]$ is the total work done by the response force. The key point of our study is computation of the response force self-consistently with the trajectory of the electron.

The charge density of the ejected electron moving along the trajectory $z_{1}(t)$ is

$$
\rho_{e}(\vec{\rho}, z, t)=-\delta^{2}(\vec{\rho}) \delta\left(z-z_{0}\right) \theta(-t)-\delta^{2}(\vec{\rho}) \delta\left[z-z_{1}(t)\right] \theta(t) .
$$

We have to compute the response force acting on the electron which at $t>0$ is at the point $\vec{R}=\left[0, z_{1}(t)\right]$. Within the linearresponse theory, the surface potential induced by an external charge density can be conventionally calculated in terms of the kernel $W\left(\vec{\rho}, z, z^{\prime}, \tau\right)$,

$$
\phi_{r}(\vec{\rho}, z, t)=\int d^{2} \vec{K} d \omega d z^{\prime} e^{i(\vec{K} \vec{\rho})-\omega t} \tilde{W}\left(\vec{K}, z, z^{\prime}, \omega\right) \tilde{\rho}_{e}\left(\vec{K}, z^{\prime}, \omega\right) .
$$

The Fourier transforms are determined as follows:

$$
W\left(\vec{\rho}, z, z^{\prime}, t\right)=\int \frac{d^{2} \vec{K} d \omega e^{i(\vec{K} \vec{\rho})-i \omega t}}{(2 \pi)^{3 / 2}} \tilde{W}\left(\vec{K}, z, z^{\prime}, \omega\right) .
$$

The response potential is a sum of two terms,

$$
\phi_{r}(\vec{\rho}, z, t)=\phi_{r 1}(\vec{\rho}, z, t)+\phi_{r 2}(\vec{\rho}, z, t),
$$

corresponding to the two densities in Eq. (2), respectively; the Fourier transforms of those densities are

$$
\begin{aligned}
& \tilde{\rho}_{1}(\vec{K}, z, \omega)=\frac{1}{(2 \pi)^{3 / 2}} \delta\left(z-z_{0}\right) \frac{i}{\omega-i 0}, \\
& \tilde{\rho}_{2}(\vec{K}, z, \omega)=-\frac{e^{i \omega \tau(z)}}{(2 \pi)^{3 / 2}} \theta\left(z-z_{0}\right) \frac{d \tau}{d z}
\end{aligned}
$$

Here the function $\tau(z)$ is the inverse to $z_{1}(\tau)$ function. We take the response kernel $\widetilde{W}\left(\vec{K}, z, z^{\prime}, \omega\right)$ within the specular reflection model, ${ }^{11}$

$$
\begin{aligned}
\tilde{W}\left(\vec{K}, z, z^{\prime}, \omega\right) & =\frac{e^{-K\left(z+z^{\prime}\right)}}{(2 \pi)^{1 / 2} K} g(K, \omega), \\
g(K, \omega) & =\frac{\epsilon_{s}(K, \omega)-1}{\epsilon_{s}(K, \omega)+1}
\end{aligned}
$$

with the simplest single surface-plasmon-pole approximation for the surface dielectric function $\epsilon_{s}(K, \omega),{ }^{8}$

$$
\epsilon_{s}(K, \omega)=\frac{\omega(\omega+i \gamma)-\alpha K-\beta K^{2}-K^{4} / 2}{\omega(\omega+i \gamma)-\omega_{p}^{2}-\alpha K-\beta K^{2}-K^{4} / 2} .
$$

The surface plasmon is determined with equation $\epsilon(K, \omega)$ $+1=0$ (Ref. 11) that leads to the dispersion law,

$$
\omega_{s p}(K)=\sqrt{\omega_{p}^{2} / 2+\alpha K+\beta K^{2}+K^{4} / 4}-i \gamma / 2
$$

$$
\simeq \frac{\omega_{p}}{\sqrt{2}}\left[1+\frac{\alpha K}{\omega_{p}^{2}}+K^{2}\left(\frac{\beta}{\omega_{p}^{2}}-\frac{\alpha^{2}}{2 \omega_{p}^{4}}\right)\right]-i \gamma / 2 .
$$

Thus, the parameters entering the dielectric function parametrization can be directly related to the surface-plasmon dispersion $\Omega_{s p}(K)=\omega_{s p}+a K+b K^{2}$,

$$
\omega_{p}=\sqrt{2} \omega_{s p}, \quad \alpha=2 a \omega_{s p}, \quad \beta=2 \omega_{s p} b+a^{2},
$$

the parameters $\omega_{s p}, \gamma, a, b$ can be measured experimentally. ${ }^{10}$

In correspondence with Eq. (4), the force acting on the electron is a sum of the two contributions, $F_{r}(t)=F_{r 1}(t)$ $+F_{r 2}(t)$

$$
\begin{aligned}
F_{r 1}(t)= & -\int_{0}^{\infty} K d K e^{-K\left(z_{1}(t)+z_{0}\right)-\gamma t / 2} \\
& \times\left[\cos D(K) t+\frac{\gamma \sin D(K) t}{2 D(K)}\right], \\
F_{r 2}(t)=\operatorname{Im} & \int_{0}^{\infty} K d K \frac{e^{-K z_{1}(t)-\gamma t / 2-i D(K) t}}{D(K)} G(K, t),
\end{aligned}
$$

where

$$
\omega_{ \pm}(K)=-i \gamma / 2 \pm D(K)
$$

$$
\begin{gathered}
D(K)=\sqrt{-\frac{\gamma^{2}}{4}+\frac{\omega_{p}^{2}}{2}+\alpha K+\beta K^{2}+K^{4} / 4}, \\
G(K, t) \equiv \frac{\omega_{p}^{2}}{2} \int_{0}^{t} d \tau e^{\gamma \tau / 2+i D(K) \tau-K z(\tau)} d \tau .
\end{gathered}
$$

The force $F_{r 1}(t)$ depends only on the current coordinate of the electron, although it is explicitly time dependent while the force $F_{r 2}$ depends on the entire previous trajectory $z_{1}(t)$.

To analyze these formulas qualitatively, we omit the dependence of $D(K)$ on $K$,

$$
\begin{gathered}
F_{r 1}(t)=-\frac{\exp (-\gamma t / 2)}{\left[z_{1}(t)+z_{0}\right]^{2}}\left(\cos D t+\frac{\gamma \sin D t}{2 D}\right), \\
F_{r 2}(t)=-\frac{\omega_{p}^{2}}{2 D} \int_{0}^{t} \frac{e^{-\gamma / 2(t-\tau)} \sin D(t-\tau)}{\left[z_{1}(t)+z_{1}(\tau)\right]^{2}} d \tau .
\end{gathered}
$$

Both formulas can be interpreted with the conventional notion of the image charge. The force $F_{r 1}$ is rather trivial: it is the force due to the oscillating and decreasing charge fixed at the initial image charge position $z=-z_{0}$. The force $F_{2 r}$ is due to the image charge which is spread over the mirror of the electron trajectory $\left[-z_{1}(t), z_{0}\right]$ with the linear charge density $\mu\left(t_{1}\right)$ depending on time $t_{1}=t-\tau$ as $\mu\left(t_{1}\right)$ $=\omega^{2} e^{-\gamma t_{1} / 2} \sin \left(D t_{1}\right) / 2 D$.

Two limit cases deserve attention. First, let us assume that the charge moves very slowly at $t>0$. In this case, $z_{1}(t)$ $\cong z_{0}$ and 


$$
F_{r 2}(t) \cong-\frac{1-e^{-\gamma t / 2}(2 D \cos D t+\gamma \sin D t) / 2 D}{4 D z_{0}^{2}} .
$$

The sum of the induced forces $F_{r 1}+F_{r 2}$ gives a conventional force in the adiabatic approximation, $-1 / 4 z_{0}^{2}$. The second case is a limit of large $t$. In this case, both $z_{1}(t)$ and $z_{1}(\tau)$ are large and the integral over time in Eq. (18) is determined by the contribution from $\tau$ close to the final time $t$. In this case, $F_{r 2}(t)$ coincides with the force given in Eq. (19) with a substitution $z_{0} \rightarrow z_{1}(t)$. Since in the large- $t$ limit, the force $F_{r 1}$ decreases exponentially, the response force on the electron becomes close to the conventional attraction to the moving image charge, $F_{r} \cong-1 / 4 z_{1}^{2}(t)$. These two limit cases correspond to the two stages of the process discussed above. The force $F_{r 1}(t)$ oscillates with the surface-plasmon frequency and decreases exponentially with the plasmon decay rate. The adiabatic image potential can be obtained in the limit $\gamma \rightarrow \infty$. Taking into account the surface-plasmon dispersion, one obtains in this limit,

$$
F_{a d}^{r}\left(z_{1}\right)=-\int_{0}^{\infty} \frac{\omega_{p}^{2} e^{-2 K z_{1}} K d K}{\omega_{p}^{2}+2 \alpha K+2 \beta K^{2}+K^{4} / 2} .
$$

The absolute value of this force is less than the conventional image potential, which follows from this equation with the surface-plasmon dispersion being neglected. The term linear in $K$ in the plasmon dispersion leads to a change in the asymptote of the response force at large $z, F_{a d}^{r} \sim-1 / 4(z$ $+\delta z)^{2}, \quad \delta z=a / \omega_{s p}$, that corresponds to the shift of the image plane into the metal. The linear term in the surface-plasmon dispersion was first discussed by Feibelman, ${ }^{12}$ who related the linear $K$ contribution in the surface-plasmon dispersion to the shape of the potential surface barrier.

Let now discuss the results of numerical computations. We consider the metal surface with the parameters of surface plasmon obtained experimentally in the case $\operatorname{Ag}(110):{ }^{10} \omega_{s p}$ $=3.70 \mathrm{eV}, \quad \gamma=0.1 \mathrm{eV}, a=1.4 \mathrm{eV} \mathrm{A}, b=1.3 \mathrm{eV} \mathrm{A}^{2}$. We consider the adsorbate, placed at $Z_{a d}=3.5$ a.u. that corresponds to the case of a heavy alkali adsorbate (e.g., Cs). The initial position of the electron is set $z_{0}=Z_{a d}+0.2$ a.u. For the relevant potentials, we take the simplest representation valid in the case of ionization of the alkali adsorbate, which donates a loosely bound electron to the metal surface, being initially adsorbed in a state of one-charged positive ion,

$$
U_{\text {core }}=-\frac{2}{\left|z-Z_{a d}\right|} ; \quad U_{\text {core }}^{\text {im }}=\frac{2}{\left|z+Z_{a d}\right|} .
$$

In Fig. 1(a), the response force acting on a charged particle moving from the point $z_{0}=3.7$ a.u. with constant velocity is plotted in comparison with the adiabatic force. One can see that at small $z$, the force is rather close to $-1 / 4 z_{0}^{2}$, being determined at the initial stage by the "frozen" image charge distribution. At large $z$, the forces converge to the corresponding adiabatic dependencies, which are different for the two types of the surface-plasmon dispersion considered. The convergence for small velocity is much faster but in all the cases, we observe oscillating dependence of the force on the $z$ coordinate.

In Fig. 1(b), the $z$ dependence of the work $A(z)$
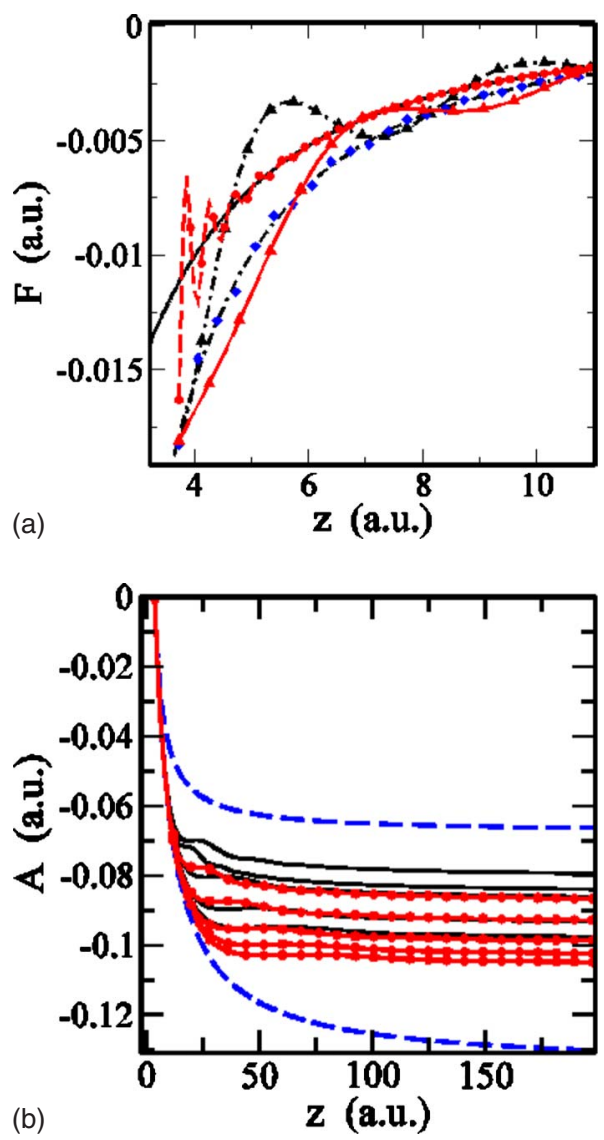

FIG. 1. (Color online) (a) The response forces $F_{r}(z)$ on the ejected electron are plotted. The solid (black) line shows the adiabatic force for the dispersive plasmon case and the dashed-dotted (black) line corresponds to the nondispersive surface-plasmon case. The dashed (red) line with circles corresponds to the response force acting on an electron moving with constant velocity $v=0.01$ a.u. and dashed-dotted line with triangles for $v=0.1$ a.u. in the case of dispersive plasmon. For the nondispersive case, diamonds (blue) corresponds $v=0.01$ a.u., solid (red) line with triangles- $v$ $=0.1$ a.u. (b) The work by the response force $F_{r}$ on the electron in dependence on the electron coordinate. The electron is ejected from the point $z_{0}=3.7$ a.u. in the case +2 -charged core. The (blue) dashed lines are $A=1 / 4 z-1 / 4 z_{0}$ (top line) and $A=1 /\left(z+z_{0}\right)$ $-1 / 2 z_{0}$ (bottom line). The solid (black) lines correspond to the nondispersive surface plasmon, the solid (red) lines with circles give the results for dispersive surface plasmon. The curves correspond to $E_{0}$ equal to $0.2,0.5,1.0,1.5$, and 2 a.u. from top to bottom.

$=\int_{z_{0}}^{z} F_{r}(z) d z$ done by the response force on the electron ejected from the adsorbate $E_{0}$ is plotted for various values of the excitation energy $E_{0}$. It is clear that at the initial stage, the work is very close to the work by the fixed initial image charge $1 /\left(z+z_{0}\right)-1 /\left(2 z_{0}\right)$. There are some weak oscillations in $A(z)$ at intermediate $z$. Then this dependence becomes very similar to the adiabatic dependence but shifted by the work performed at the initial stage. The intermediate region increases with increase in $E_{0}$, as well as the work accumulated in this region.

In Fig. 2, the total work $A(\infty)$ is plotted as a function of the excitation energy $E_{0}$. The total work is sensitive to the dispersion of the surface plasmon. It changes in electron volt 


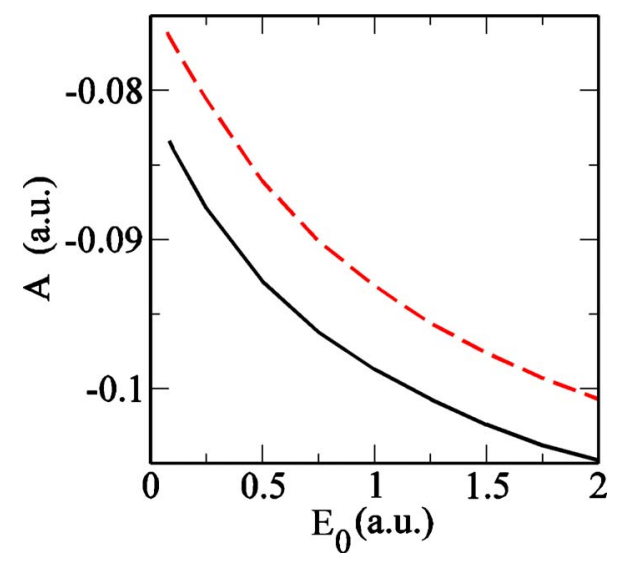

FIG. 2. (Color online) The work by the response force in dependence on the excitation energy $E_{0}$ is plotted. The dashed (red) line corresponds to the surface plasmon with dispersion and the solid (black) corresponds to the nondispersive surface plasmon.

range when the excitation energy changes in $10 \mathrm{eV}$ range. This dependence can be observed with the modern sources of the extreme ultraviolet-soft $\mathrm{x}$ radiation.

The minimum value of the energy $E_{0}$ which is necessary for ionization depends on the dispersion of the surface plasmon. For the adiabatic potential, the threshold energy is $E_{t h r}^{a d}=0.0676$ a.u. $=1.84 \mathrm{eV}$, in the case of the nondispersive surface plasmon $E_{t h r}^{\text {non-disp }}=0.0834$ a.u. $=2.27 \mathrm{eV}$, and in the case of the dispersive plasmon $E_{t h r}^{d i s p}=0.0767$ a.u. $=2.09 \mathrm{eV}$.

Summarizing, we have described a general phenomenon in dynamical relaxation of the electron system of a metal caused by a fast electron ejected from an adsorbate perpendicular to the metal surface. The conventional theory of metal surface response based on the idea of image charge presumes the motion of a charged particle to be adiabatically slow. This assumption is obviously broken in the case of photoionization of the internal shell of an adsorbate. In this case, the ejected electron moves quite fast and at the first stage of the process, the charge distribution in the metal remains frozen. The force from the metal response charge distribution can be universally represented as a sum of two contributions. The first contribution is due to the oscillating and decreasing initial image charge fixed at the mirror point of the initial position of the electron. The second force can be interpreted as a force from the image charge spread along the mirror line to the electron trajectory with oscillating and decreasing linear charge density. The work done by the dynamical response force on the ejected electron is larger than that obtained within the adiabatic assumption. The additional energy loss depends on the ejection energy of the ionized electron and this dependence can be observed experimentally.

P.M.E. acknowledges partial support from the University of the Basque Country (Grant No. 9/UPV 00206.215-13639/ 2001), the Basque Universitate eta Ikerketa Saila, and the Spanish Ministerio de Education y Ciencia (MEC) (Grants No. FIS 2004-06490-C03-01 and No. CSD2006-53). We are thankful J.-P. Gauyacq, A. G. Borisov, V. M. Silkin, and N. M. Kabachnik for useful discussions.
${ }^{1}$ F. Krausz and M. Ivanov, Rev. Mod. Phys. 81, 163 (2009).

${ }^{2}$ M. Hentschel, R. Kienberger, C. Spielmann, G. A. Reider, N. Milosevic, T. Brabec, P. Corkum, U. Heinzmann, M. Drescher, and F. Krausz, Nature (London) 414, 509 (2001).

${ }^{3}$ A. K. Kazansky and N. M. Kabachnik, J. Phys. B 40, 2163 (2007); 39, 5173 (2006); 40, 3413 (2007).

${ }^{4}$ A. L. Cavalieri, N. Muller, T. Uphues, V. Yakovlev, A. Baltuska, B. Horvath, B. Schmidt, L. Blumel, R. Holzwarth, S. Hendel, M. Drescher, U. Kleineberg, P. M. Echenique, R. Kienberger, F. Krausz, and U. Heinzmann, Nature (London) 449, 1029 (2007).

${ }^{5}$ A. K. Kazansky and P. M. Echenique, Phys. Rev. Lett. 102, 177401 (2009).
${ }^{6}$ C. Lemell, B. Solleder, K. Tökési, and J. Burgdörfer, Phys. Rev. A 79, 062901 (2009).

${ }^{7}$ J. D. Jackson, Classical Electrodynamics (Wiley, New York, 1962).

${ }^{8}$ P. M. Echenique, V. H. Ponce, M. F. Uranga, and F. J. G. de Abajo, Nucl. Instrum. Methods Phys. Res. B 96, 583 (1995).

${ }^{9}$ J. M. Pitarke, V. M. Silkin, E. V. Chulkov, and P. M. Echenique, Rep. Prog. Phys. 70, 1 (2007).

${ }^{10}$ M. Rocca, F. Biggio, and U. Valbusa, Phys. Rev. B 42, 2835 (1990).

${ }^{11}$ R. H. Ritchie and A. L. Marusak, Surf. Sci. 4, 234 (1966).

${ }^{12}$ P. J. Feibelman, Phys. Rev. Lett. 30, 975 (1973). 